\title{
A numerical study on the coupled hydro-mechanical behaviour of compacted bentonite
}

\author{
Jose A. Bosch ${ }^{1, *}$, Alessio Ferrari ${ }^{1}$, and Lyesse Laloui ${ }^{1}$ \\ ${ }^{1}$ Swiss Federal Institute of Technology in Lausanne, Lausanne, Switzerland
}

\begin{abstract}
Understanding the mechanical behaviour of compacted bentonite upon re-saturation is of outmost importance in most designs of nuclear waste disposal repositories. The behaviour of bentonite is characterized by its stress-path dependency and it is typically interpreted on the basis of its microstructural interactions. Up to now, effective stress-based models have had limited success in reproducing consistently the main responses. Here a recently proposed model for the modelling of volumetric behaviour of compacted bentonites is extended to triaxial stress states. The model is formulated using a conventional effective stress expression and the degree of saturation. Because these two variables are directly related to the water retention, a suitable formulation for bentonites is used. The resulting equations are characterised by a high degree of hydromechanical couplings and a low number of material parameters, which can be obtained on the basis of wellestablished laboratory procedures. In order to demonstrate its capabilities, the model is used to simulate the behaviour of MX- 80 bentonite for several stress paths under oedometric conditions. The emphasis is put on the process of parameter determination. The predictive capabilities of the model are also highlighted.
\end{abstract}

\section{Introduction}

One of the main challenges in the analysis of underground nuclear waste repositories that use bentonite as a sealing material, is the long-term prediction of dry density evolution and distribution of the bentonite. In this regard, it is paramount the use of numerical models that can be calibrated on a well-defined basis and preferably with measurable properties, while being able to describe and predict the behaviour of bentonite satisfactorily. Such behaviour entails high swelling strains, high swelling pressure and stress path dependency [1].

This paper reports on-going work in the constitutive modelling of bentonite behaviour. A hydro-mechanical constitutive model is presented, calibrated and validated against experimental data that involves relevant hydromechanical stress paths. The model is based on the recently developed volumetric model presented in Bosch et al. [2] which is formulated in terms of a single effective stress and the degree of saturation. This model here is extended to triaxial stress states using the Modified Cam Clay yield surface, an associated flow rule and a constant Poisson ratio. Although the model equations are highly coupled, they are relatively simple, and especially suitable for its numerical implementation, due to the smooth and natural transition between unsaturated and saturated states. The calibration procedure of all material parameters is shown for MX-80 bentonite, demonstrating that the determination of parameters can be done in a robust way on the basis of quantitative data from laboratory tests. Once the material parameters have been established, the predictive capabilities of the model are evaluated with wetting under constant load tests that led to both collapse and swelling strains.

\section{Numerical models}

The model is formulated in terms of the following triaxial stress variables:

$$
p^{\prime}=\frac{1}{3}\left(\sigma_{a}^{\prime}+2 \sigma_{r}^{\prime}\right), \quad q=\sigma_{a}^{\prime}-\sigma_{r}^{\prime}
$$

and the strain variables:

$$
\epsilon_{v}=\epsilon_{a}+2 \epsilon_{r}, \quad \epsilon_{q}=2 / 3\left(\epsilon_{a}-\epsilon_{r}\right)
$$

where $\sigma^{\prime}$ stands for the effective stress, $\epsilon$ for the total strain and subscripts $a, r$ refer to the axial and radial directions, respectively. The sign convention is positive for compressive stress and contraction strains and negative for tensile stress and expansion strains.

The constitutive equations are formulated according to the theory of elasto-plasticity whereby an explicit division between elastic and plastic (irreversible) strains is considered:

$$
\mathrm{d} \boldsymbol{\epsilon}=\mathrm{d} \boldsymbol{\epsilon}^{e}+\mathrm{d} \epsilon^{p}
$$

where $\boldsymbol{\epsilon}$ is the (total) strain tensor and superscripts $e, p$ stand for elastic and plastic strains respectively.

\footnotetext{
* Corresponding author: jose.boschllufriu@epfl.ch
} 
Elastic strains are due to changes in the generalised effective stress $\boldsymbol{\sigma}^{\prime}$, in accordance with Nuth and Laloui [3]:

$$
\boldsymbol{\sigma}^{\prime}=\boldsymbol{\sigma}-\left[p_{a}-s S_{r}\right] \mathbf{I}
$$

where $\boldsymbol{\sigma}$ is the total stress, $p_{a}$ is the pore air pressure, $s=$ $p_{a}-p_{w}$ is the matric suction, $p_{w}$ is the pore water pressure and $S_{r}$ is the degree of saturation. Eq. 4 is a particular form of the expression suggested by Bishop [4].

Elastic strains result from the nonlinear relations

$$
\mathrm{d} \epsilon_{v}^{e}=\frac{p^{\prime}}{\kappa} \mathrm{d} p^{\prime}, \quad \mathrm{d} \epsilon_{q}^{e}=\frac{9(1-2 v)}{2(1+v)} \frac{p^{\prime}}{\kappa} \mathrm{d} q
$$

where $v$ is the Poisson ratio and $\kappa$ is a material parameter defining the elastic compressibility.

Plastic strains develop when the yield condition $f_{Y}=$ 0 is met, with $f_{Y}$ being the yield surface. The Modified Cam Clay yield surface and an associated flow rule are used:

$$
f_{Y}=q^{2}-M^{2}\left(p_{Y}^{\prime}-p^{\prime}\right) p^{\prime}=0
$$

where $M$ defines the stress ratio at critical state and $p_{Y}^{\prime}$ is the yield pressure.

Similarly to [5] the yield pressure, $p_{Y}^{\prime}$ evolves with plastic deformation and degree of saturation according to the expression

$$
\frac{p_{Y}^{\prime}}{p^{\prime}{ }_{r}}=\left(\frac{p_{Y_{S}}^{\prime}}{p^{\prime}{ }_{r}}\right)^{\frac{\lambda_{s}-\kappa}{\lambda\left(S_{r}\right)-\kappa}}
$$

where $p^{\prime}{ }_{r}$ is a reference stress (generally set to values around $1 \mathrm{~Pa}), \lambda_{s}$ defines the elasto-plastic compressibility for saturated states; $\lambda\left(S_{r}\right)$ is a function expressing the evolution of elasto-plastic compressibility with the degree of saturation; and $p_{Y S}^{\prime}$ is the corresponding yield pressure at saturated state, which depends on the hardening law:

$$
\frac{\mathrm{d} p_{Y S}^{\prime}}{p_{Y S}^{\prime}}=\frac{\mathrm{d} \epsilon_{v}^{p}}{\lambda_{s}-\kappa}
$$

The following expression is used for $\lambda\left(S_{r}\right)$ :

$$
\lambda\left(S_{r}\right)=\lambda_{s}-r\left(\lambda_{s}-\kappa\right)\left(1-S_{r}^{\zeta}\right)^{\xi}
$$

where $r, \zeta$ and $\xi$ are parameters defining the evolution of compressibility between dry and saturated states.

The degree of saturation is computed as the ratio between water ratio $e_{w}$ (ratio of water volume with respect to volume of solids) and void ratio $e$ :

$$
S_{r}=\frac{e_{w}}{e}
$$

The water retention model brings together an adsorption model and a capillary model [2] following ideas similar to [6]. $e_{w}$ is divided into capillary water ratio, $e_{w, c}$ (volume of non-adsorbed water with respect to volume of solids) and adsorbed water, $e_{w, a}$ (volume of adsorbed water with respect to volume of solids):

$$
e_{w}=e_{w, c}+e_{w, a}
$$

The evolution of capillary water ratio $e_{w, c}$ is modelled with the expression proposed by van Genuchten [7] and modified by [8]:

$$
e_{w, c}=\left(e-e_{w, a}\right)\left\{1+\left[a\left(e-e_{w, a}\right)^{b} s\right]^{n}\right\}^{1 / n-1}
$$

where $n, a$ and $b$ are material parameters. $e_{w, a}$ is a function of relative humidity, $R H$, following a Freundlich isotherm [9] and expressed in terms of $s$ by means of Kelvin's law:

$$
e_{w, a}=e_{w, a}^{C}\left[\exp \left(-\frac{M_{w}}{\rho_{w, a} R T} s\right)\right]^{1 / m}
$$

where $\rho_{w, a}$ is the density of adsorbed water, $R=8.314$ $\mathrm{J} / \mathrm{mol} \mathrm{K}, R$ is the gas constant, $M_{w}$ is the molar volume of water, $T$ is the temperature in Kelvin degrees, $e_{w, a}^{C}$ is the adsorption capacity parameter, and $m$ is a material parameter.

\section{Model application}

\subsection{Determination of parameters}

In this section, a procedure for the determination of model parameters is suggested. Although robust, it is not a unique procedure, especially for the parameters that define the elasto-plastic compressibility under unsaturated states, i.e. Eq. (9). Here the unsaturated compressibility will be determined on the basis of a swelling pressure test, although alternatively these can be calibrated using compression under unsaturated states, as shown in [2]. Therefore, alternative procedures of calibration can be used depending on the availability of experimental data. The model is applied to compacted (block) MX-80 bentonite, given the extensive results on the behaviour of this material reported in the literature.

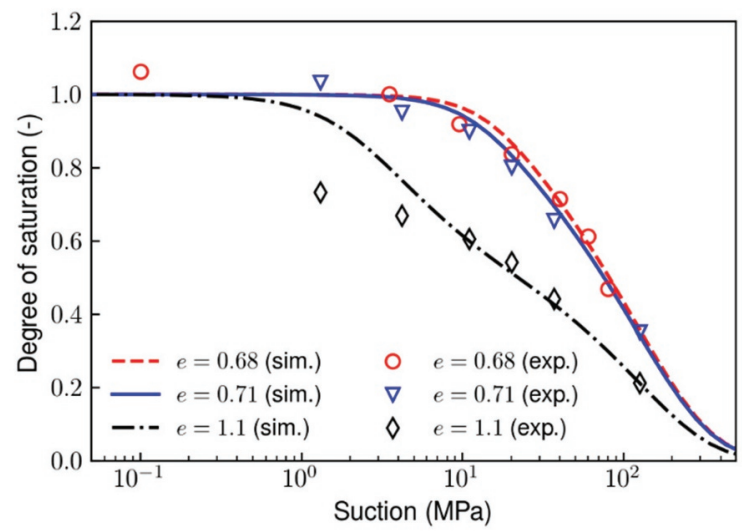

Figure 1. Water retention calibration with experimental data (reported by [10] and [11]) on wetting paths for MX-80 bentonite under constant volume. 
Table 1. Water retention parameters for the compacted MX80 bentonite.

\begin{tabular}{|c|c|}
\hline Parameter & Value \\
\hline$a$ & $0.9 \mathrm{MPa}^{-1}$ \\
\hline$b$ & 1.5 \\
\hline$n$ & 1.8 \\
\hline$m$ & 1.0 \\
\hline$e_{w, a}^{C}$ & 0.55 \\
\hline
\end{tabular}

First, the model is calibrated based on water retention properties, which is a fundamental relationship determining the model performance, due to the use of the degree of saturation as a constitutive variable. Three or more tests of water retention are preferable if only constant volume results are available, whereas one test performed under free volume conditions in combination with a constant volume test can be used to determine quite accurately all the water retention parameters.

Conveniently, the water retention behaviour of the MX-80 bentonite has been extensively tested. Figure 1 shows the fit of the water retention model to the experimental data reported in [10] and [11]. The values used for each parameter are summarised in Table 1.

Note that the degree of saturation from the experimental data was computed using a water density of $1 \mathrm{Mg} / \mathrm{m}^{3}$, which resulted in degree of saturation higher than 1 for the samples at $e=0.68$ at low suctions. For the sake of conciseness, in the present case adsorbed water density is also taken as $1 \mathrm{Mg} / \mathrm{m}^{3}$.

Once the water retention model is calibrated, the evolution of effective stress can be computed and therefore the calibration of the mechanical parameters can be done. Figure 2 shows the calibration of the parameters $\kappa$ and $\lambda_{s}$ on the basis of isotropic compression and unloading at saturated states reported by [12]. The stress ratio at critical state is set as $M=0.37$ based on triaxial tests reported by [13] on compacted saturated bentonite. The Poisson ratio is set as $v=0.25$.

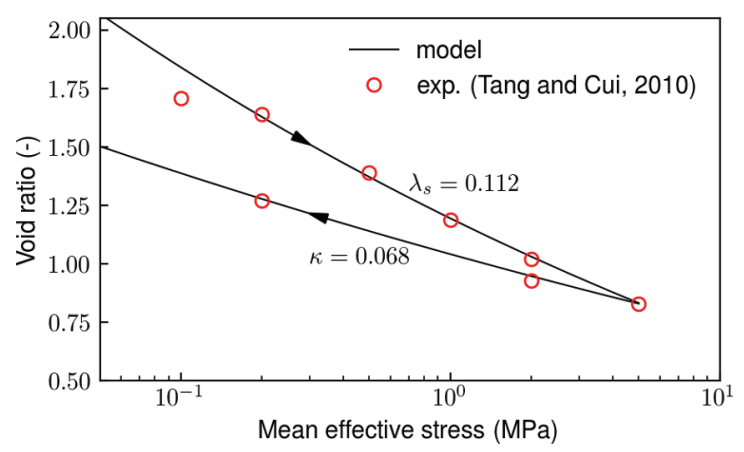

Figure 2. Calibration of $\lambda_{s}$ and $\kappa$ with isotropic compression and unloading tests on MX80 at saturated states. Data from [12].

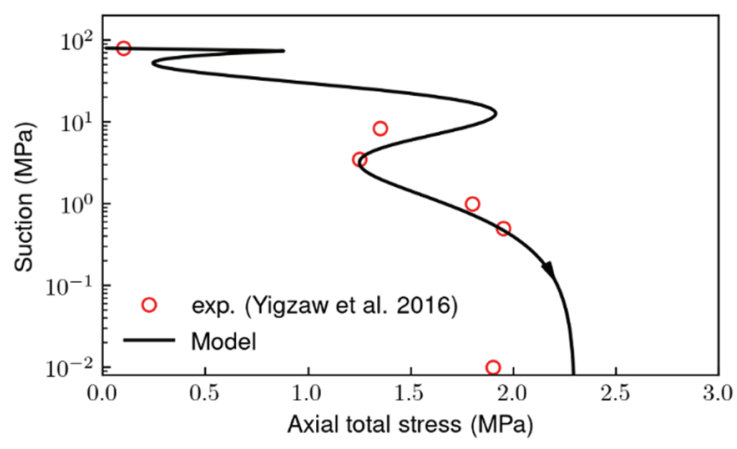

Figure 3. Calibration of the unsaturated elasto-plastic compressibility parameters: $\zeta, \xi$ and $r$ with suction controlled swelling pressure test on compacted MX80 with $\mathrm{e}=0.97$ data from [14].

Table 2. Material parameters for the MX80 compacted bentonite.

\begin{tabular}{|c|c|}
\hline Parameter & Value \\
\hline$\kappa$ & 0.068 \\
\hline$v$ & 0.25 \\
\hline$\lambda_{s}$ & 0.112 \\
\hline$M$ & 0.37 \\
\hline$p_{r}^{\prime}$ & $1 \mathrm{~Pa}$ \\
\hline$r$ & 0.45 \\
\hline$\zeta$ & 1.5 \\
\hline$\xi$ & 0.77 \\
\hline
\end{tabular}

Yigzaw et al. [14] performed a set of constant volume (at void ratio of $e=0.97$ ) swelling pressure tests in which suction was controlled. These tests provide valuable insight regarding the compressibility under unsaturated conditions. Indeed, despite that the volume is constant during these tests, an important rearrangement of the fabric of the material occurs over the course of the saturation process (see for instance references [15] and [16]), which could be irreversible (Seiphoori et al. [17]). In the present modelling approach, this is interpreted as a plastic process, which depends on the relationship between the elastic compressibility $\kappa$, and the elastoplastic compressibility $\lambda\left(S_{r}\right)$, along the different saturation states. Hence, the evolution of swelling pressure with suction, provides a means to calibrate the parameters accounting for the unsaturated elasto-plastic compressibility.

Figure 3 shows the model response. In order to achieve a reasonable fit with the data, the yield limit is reached at relatively high suctions after an axial pressure of $1 \mathrm{MPa}$ develops. The subsequent development of swelling pressure is in agreement with the experimental 
data. This not only depends on the mechanical parameters, but it is also strongly dependent on the water retention curve through the effective stress formulation (eq. 4). As a result, in the current calibration, the set of hydromechanical parameters leads to a non-linear response with two distinct peaks as suction decreases. Hence the importance of a good calibration of the water retention curve prior to proceed to the calibration of the mechanical model. The complete set of mechanical parameters is summarised in Table 2 .

\subsection{Model predictions}

In order to demonstrate the predictive potential of the model, three tests reported by [14], consisting in suction decrease under constant load, are simulated. These stress paths were not considered in the calibration procedure and therefore they provide a valuable example to assess predictive capabilities. Three tests are simulated; starting from the initial state at a void ratio of $e=0.97$ and $s=80 \mathrm{MPa}$, compression stages up to axial stress of 0.25 $\mathrm{MPa}, 1 \mathrm{MPa}$ and $2 \mathrm{MPa}$ are performed before imposing a decrease of suction to $s=0 \mathrm{MPa}$. Radial strains are prevented in all cases.

Figure 4 shows the modelling predictions compared to the experimental results obtained by [14], it is observed that the predicted evolution of void ratio match fairly well with those measured experimentally, hence giving confidence in the material parameters determined with swelling pressure tests. It is remarkable the smooth transition to the saturated states and the prediction of both collapse and swelling upon wetting depending on the vertical load. Note that the swelling strains stabilise once the suction becomes lower than the value of vertical load. This is a result of the logarithmic relationship between effective stress and volumetric strains. Indeed, when the value of suction drops below that of the vertical load, the effective stress tends to the total stress, reaching thus a state of equilibrium that agrees well with the experimental data. Because the tests were performed under oedometric conditions, radial stress increased upon wetting in order to keep null radial displacements. Therefore, the final state at zero suction does not necessarily correspond to a mean effective stress equal to the vertical load.

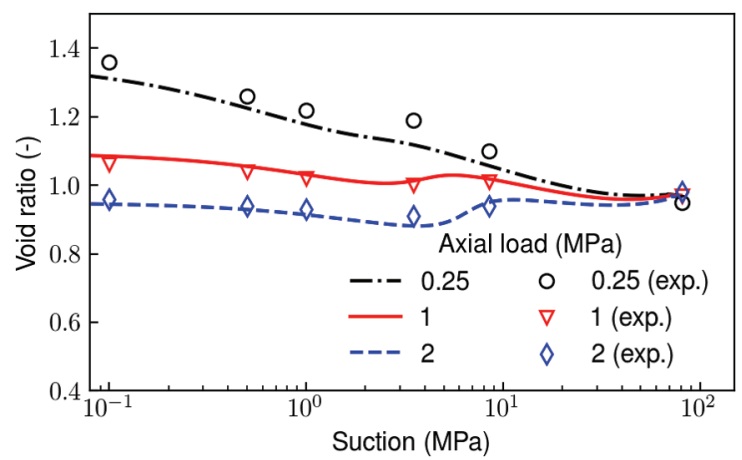

Figure 4. Model prediction of void ratio evolution upon wetting under constant load. Experimental data on MX80 compacted bentonite reported by Yigzaw et al. (2016) is also represented for comparison purposes.


Figure 5. Interpretation of bentonite behaviour upon wetting under different vertical stress in terms of effective stress and degree of saturation. As a reference, the initial yield, the normal compression line at saturated state and the purely elastic line from the initial state are also shown.

To understand the differences between the wetting under constant volume (swelling pressure) and the wetting under constant load, the stress paths computed by the model in terms of constitutive stresses (effective stress and degree of saturation) are reproduced in Figure 5. It is observed that, as in the case of constant volume wetting, the purely elastic behaviour is restricted to the first part of the tests. As wetting proceeds beyond that point and the degree of saturation increases, the material undergoes plastic strains, the magnitude of which depends on the load to which it is subjected. In this regard, the evolution of the degree of saturation largely determines this extent of plastic strains. Indeed, when bentonite densifies, the degree of saturation increases at a higher rate than in the cases in which the bentonite undergoes swelling for the same suction range. If the vertical load is low enough, plastic strains do not exceed elastic strains and therefore no wetting-collapse is predicted. Note also, that the swelling pressure at equilibrium (when suction tends to 0 ) corresponds to the normal compression line, defined by $\lambda$, when is projected in the plane $\left(p^{\prime}-e\right)$.

\section{Conclusions}

A complete hydro-mechanical constitutive model, intended to be used to analyse bentonite barriers in nuclear waste repositories, has been described in this paper. Two of the main advantages of the model have been demonstrated, which are a robust procedure for the calibration of material parameters and the predictive capabilities. This is achieved by using a proper water retention formulation and a constitutive framework formulated in terms of an effective stress and the degree 
of saturation. As a result of the high degree of hydromechanical coupling, the number of equations and parameters necessary is significantly lower with respect to those required in a framework with independent stress variables or using a double porosity formulation.

To demonstrate some essential features, the model has been used to reproduce the response of MX80 bentonite for different stress paths starting from ascompacted conditions. It has been shown that each parameter can be calibrated on a well-defined basis, avoiding ambiguity, non-uniqueness of solutions and thus providing confidence when predictive modelling is performed, especially involving complex stress paths not considered in the calibration process. Of particular relevance is its suitability for Finite Element analysis, due to its compact mathematical formulation and the seamless transition to saturated states from as-compacted conditions.

This work has received funding from the Euratom research and training programme 2014-2018 under grant agreement No 745942 .

\section{References}

[1] A. Gens and E. E. Alonso. A framework for the behaviour of unsaturated expansive clays. Canadian Geotechnical journal. 29, 1013 (1992).

[2] J. A. Bosch, A. Ferrari and L. Laloui. On the coupling between water retention and volume change of compacted bentonite (under review).

[3] M. Nuth and L. Laloui. Effective stress concept in unsaturated soils. International Journal for Numerical and Analytical Methods in Geomechanics. 32, 771 (2008).

[4] A.W. Bishop. The effective stress principle. Teknisk ukeblad. 39, 859 (1959).

[5] A.N. Zhou, D. Sheng, S.W. Sloan, A. Gens. Intepretation of unsaturated soil behaviour in the stress-saturation space I: volume change and water retention behaviour. Computers and Geotechnics. 43, 178 (2012).

[6] A. Revil, N. Lu. Unified water isotherms for clayey porous materials. Water Resources Research. 49, 5685 (2013).

[7] Van Genuchten. A closed-form equation for predicting the hydraulic conductivity of unsaturated soils. Soil science Society of America Journal. 44, 892 (1980).

[8] D. Gallipoli, S. Wheeler, M. Karstunen. Modelling the variation of the degree of saturation in a deformable unsaturated soil. Géotechnique. 53, 105 (2003).

[9] H. Freundlich. Kapillarchemie eine darstellung der chemie der kolloide und verwandter geniete. akademische Verlagsgesellschaft. (1909).

[10] M.V. Villar. MX80 bentonite termal-hydromechanical characterisation. Informes Técnicos CIEMAT. 1053, 39 (2005).

[11] A.M. Tang and Y.J. Cui. Journal of Rock Mechanics and Geotechnical Engineering. Effects of mineralogy on thermo-hydro-mechanical parameters of MX80 bentonite. 2, 91-96 (2010).

[12] A.M. Tang and Y.J. Cui. Journal of Rock Mechanics and Geotechnical Engineering., 2 (1): 39-43 (2010).

[13] Dueck and Nilsson. SKB technical report TR-10-55 (2011).

[14] Z. G. Yigzaw, O. Cuisiner, L. Massat, F. Masrouri. Role of different suction components on swelling behaviour of compacted bentonites. Applied Clay Science. 120, 81 (2016).

[15] A. Ferrari, A. Seiphoori, Ruedi, J., L. Laloui. Shotclay assessment of the hydro-mechanical behaviour. Engineering geology, 173, pp.10-18 (2014).

[16] D. Manca, A. Ferrari, L. Laloui. Fabric evolution and the related swelling behaviour of a sand/bentonite mixture upon hydro-chemo-mechanical loadings. Géotechnique. 66, 17 (2016).

[17] A. Seiphoori, A. Ferrari, L. Laloui. Water retention behaviour and microstructural evolution of MX80 bentonite during wetting and drying cycles. Géotechnique. 64, 721 (2014). 